\title{
Control natural de plagas en cultivos mediterráneos
}

\section{J. Quinto ${ }^{1}$, A. Pineda ${ }^{1,2}, M^{a}$ Á. Marcos-García ${ }^{1}$}

i Centro Iberoamericano de la Biodiversidad CiBiO, Universidad de Alicante, España 2 Laboratory of Entomology, Wageningen University, Holanda

\section{ABSTRACT}

The spontaneous presence of natural enemies in crops represents one of the best allied to the Integrated Pest Management programs. The habitat management includes preventive measures based on close knowledge of the functioning of agroecosystems, performing actions upon pests and their enemies. These actions carried out on the habitats both in crops and in the non-cultivated areas, are aimed to conserve and enhance the populations of natural enemies. These preventive methods preserve the biodiversity of pest natural enemies in agroecosystems, using suitable management practises in agreement with ecological agriculture, and maintaining a high number of species, both predators and parasitoids.

\section{KEYWORDS}

Enemigos naturales, cultivos protegidos,

\section{INTRODUCCIÓN}

La protección fitosanitaria supone desde hace algún tiempo uno de los principales problemas con los que se enfrenta la producción de hortícolas en los cultivos del sureste Ibérico. El uso de plaguicidas no selectivos en el espacio y en el tiempo supone el factor que más reduce la efectividad de los enemigos naturales como agentes controladores de las plagas.

La evolución de la legislación que regula la agricultura Europea a través de la Política Agrícola Común (PAC.) ha modificado en gran medida el modelo agrícola tradicional, limitando el uso de productos químicos y estableciendo programas de control basados en el Manejo Integrado de Plagas (MIP), que comprenden la aplicación de dos o más técnicas de control para mantener una plaga por debajo del umbral económico, con el menor impacto para el medio ambiente (STERN et al., 1959).

El control integrado de plagas está basado en distintas estrategias que dan énfasis al control biológico, aunque además pueden incluir estrategias de control cultural, la utilización de variedades resistentes de plagas, el uso de plaguicidas de origen natural e incluso de síntesis. Una buena parte de programas de control biológico de plagas se basan en el "control biológico natural", es decir, en la presencia espontánea de los enemigos naturales en los 
cultivos, por lo que resulta muy importante que éstos encuentren un hábitat favorable para el desarrollo de sus poblaciones.

El manejo o gestión del hábitat es una forma de control de plagas denominada "control biológico por conservación", que consiste en la modificación de los hábitats para mejorar la disponibilidad de los recursos requeridos por los enemigos naturales y alcanzar un óptimo rendimiento de los mismos (LANDIS et al., 2000). La presencia de un mosaico rico de especies vegetales en forma de policultivos, sistemas diversificados de cultivo-malezas, cultivos de invernadero, etc., permiten conservar una fauna rica de enemigos naturales al asegurarles una serie de requisitos ecológicos, como es un mayor acceso a huéspedes alternativos o a recursos alimenticios como polen y néctar, y hábitats para la hibernación y la oviposición (COLLEY \& LUNA, 2000).

Hay tres grandes grupos de enemigos naturales de las especies plaga: depredadores, parasitoides y patógenos. La disponibilidad de recursos alimenticios alternativos puede mejorar el crecimiento, supervivencia, desarrollo y reproducción de ciertos depredadores y parasitoides (LANDIS et al., 2000; BERNDT y WRATTEN, 2005). Favoreciendo el desarrollo de las poblaciones naturales de estos insectos auxiliares, podemos evitar el tener que poner en marcha acciones costosas de su potenciación y evitar la introducción de especies alóctonas cuyas consecuencias ambientales no son siempre fácilmente predecibles.

El sureste ibérico posee una gran tradición citrícola y hortícola debido a su clima idóneo para la agricultura. Como consecuencia, apenas existe vegetación natural y abundan diversos tipos de cultivos que se rigen mayoritariamente bajo sistemas de producción integrada, y donde predomina el manejo del hábitat, el control cultural y el control biológico. La presencia de un complejo mosaico de cultivos (Fig. 1) dota a estos agroecosistemas diversificados de una rica entomofauna auxiliar, que de forma natural regula en gran medida la proliferación de las plagas de insectos, así como los daños originados por las mismas.

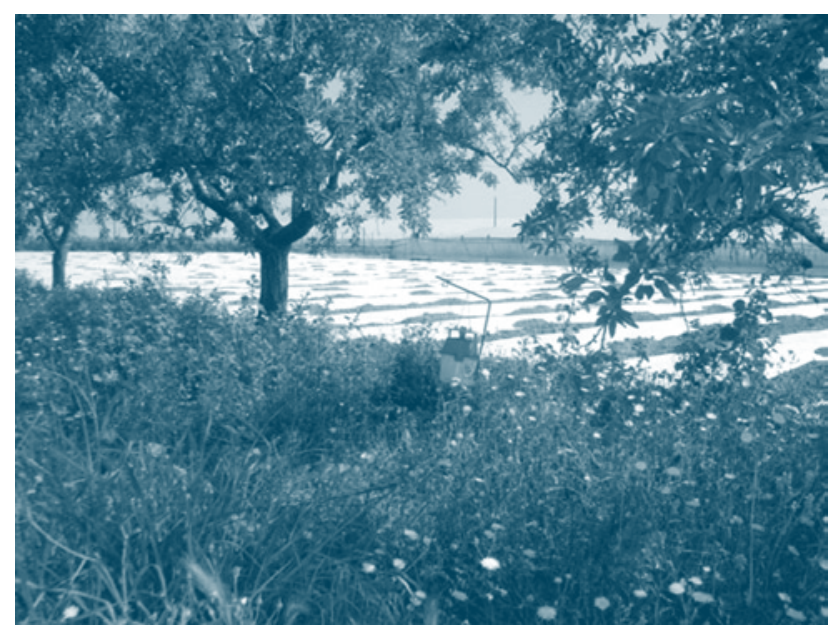

Figura 1. Agroecosistema mediterráneo típico con vegetación natural en los márgenes (Pilar de la Horadada). Foto J. Quinto

\section{MATERIALES Y MÉTODOS}

Este estudio fue llevado a cabo en Pilar de la Horadada (Alicante), de enero a julio durante los años 2006 y 2009, coincidiendo con el periodo en el que se da una mayor producción de hortícolas en esta área del sureste de España. A partir de la utilización de trampas Malaise modificadas se evaluó la diversidad de enemigos naturales de hemípteros esternorrincos (p.e. pulgones y mosca blanca) presentes en cuatro localidades de características similares, separadas entre sí por más de un kilómetro. Del mismo modo se identificaron las principales especies de hemípteros esternorrincos presentes en el medio.

Para la identificación de las especies de hemípteros esternorrincos se utilizaron las claves REMAUDIERE (1990), BLACKMAN \& EASTOP (2000). Los parasitoides de pulgones fueron identificados a partir de las claves de STARY (1976), PENNACHIO (1989) y ÖLMEZ et al. (2003). Los coccinélidos fueron identificados mediante las claves de PLAZA (1979) y a partir de la colección de comparación de la Colección Entomológica de la Universidad de Alicante (CEUA). Para la identificación de los sírfidos de utilizaron las claves de VAN DER VEEN, M. (2004). Para identificación del material recolectado se recurrió a la revisión por parte de los especialistas de cada grupo de insectos. 


\section{RESULTADOS}

Las especies plaga de hemípteros esternorrincos registradas a lo largo de los dos años de muestreo fueron principalmente los pulgones (Aphididae) Myzus persicae (Sulzer, 1776), Aulacorthum solani (Kaltenbach, 1843), Aphis gossypii (Glover, 1877) y Macrosiphum euphorbiae (Thomas, 1878); y en menor medida las "moscas blancas" (Aleyrodidae) Bemisia tabaci (Gennadius, 1889) y Trialeurodes vaporariorum (Westwood, 1856).

La riqueza de enemigos naturales registradas a lo largo de los años 2006 y 2009 asciende a un total de 48 especies: 31 de depredadores: Muscidae, Syrphidae y Cecidomyiidae (Diptera), Coccinellidae (Coleoptera), y Chrysopidae (Neuroptera); y 17 de parasitoides: Aphelinidae y Braconidae: Aphidiinae (Hymenoptera).

\section{DEPREDADORES}

\section{DIPTERA}

\section{Muscidae}

- Coenosia attenuata Stein, 1903: 2006, 2009

Cecidomyiidae

- Aphidoletes aphidimyza (Rondani, 1847): 2006, 2009

Syrphidae

- Chrysotoxum intermedium (Meigen, 1822): 2006, 2009

- Episyrphus balteatus (De Geer, 1776): 2006, 2009

- Eupeodes corollae (Fabricius, 1794): 2006, 2009 (Fig. 2)

- Paragus bicolor (Fabricius, 1794): 2006, 2009

- Paragus haemorrhous Meigen, 1822: 2006, 2009

- Paragus quadrifasciatus Meigen, 1822: 2006, 2009

- Scaeva albomaculata (Macquart, 1842): 2006

- Scaeva pyrastri (Linnaeus, 1758): 2006
- Sphaerophoria rueppellii (Wiedemann, 1830): 2006, 2009 (Fig. 3)

- Sphaerophoria scripta (Linnaeus, 1758): 2006, 2009

- Syrphus ribesii (Linnaeus, 1758): 2006

- Xanthogramma marginale (Loew, 1854): 2009

- Xanthogramma pedissequum (Harris, 1782): 2006

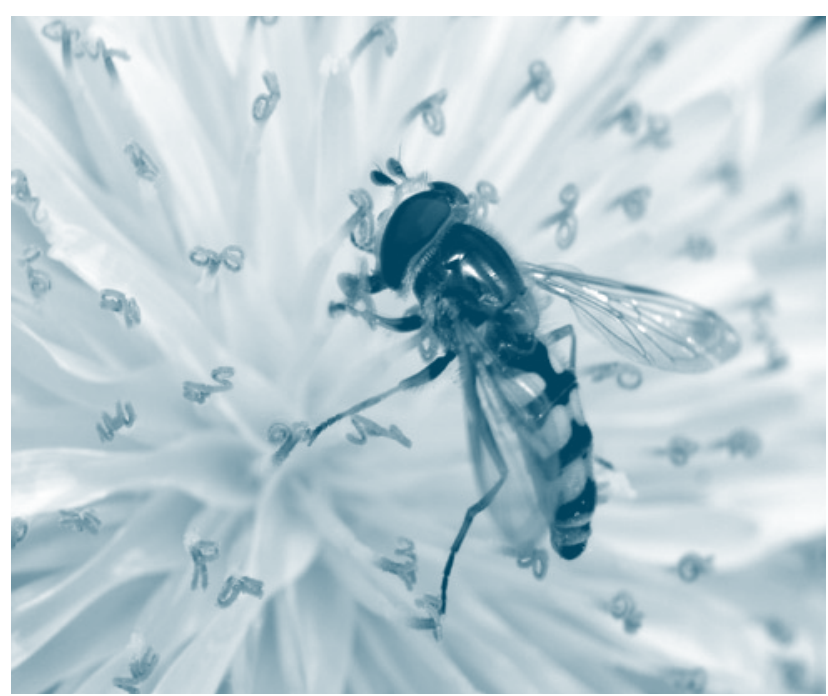

Figura 2. Eupeodes corollae. Foto J. Ordóńez

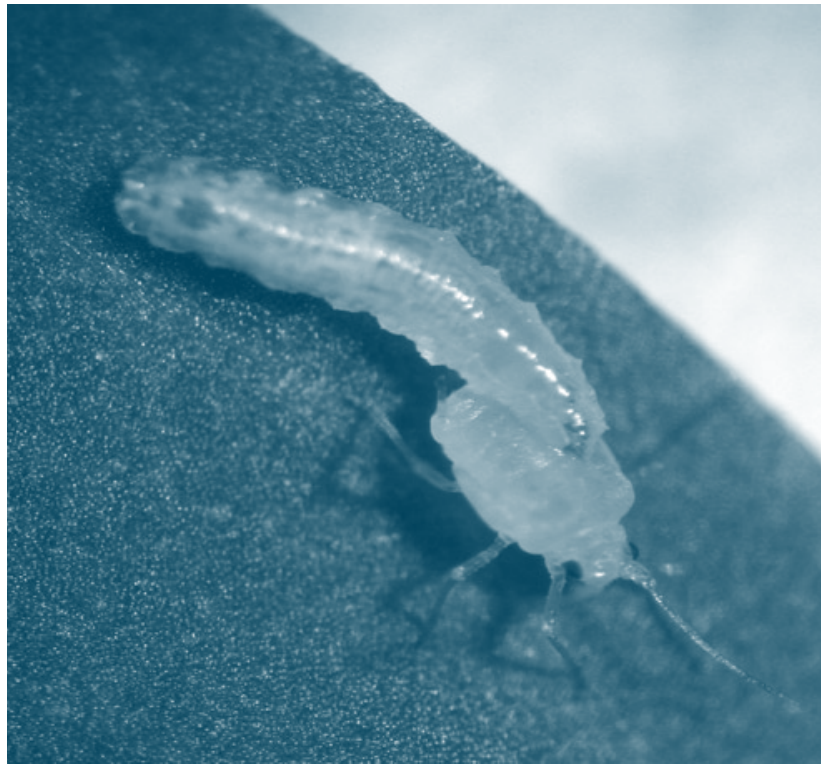

Figura 3. Larva de Sphaerophoria rueppellii depredando pulgón. Foto R. Amorós 


\section{NEUROPTERA}

Chrysopidae

- Chrysopa septempunctata (Wesmael, 1841): 2006

- Chrysoperla canea (Stephens, 1836): 2006, 2009

\section{COLEOPTERA}

Coccinellidae

- Adalia bipunctata (Linnaeus, 1758): 2006

- Clitostethus arcuatus (Rossi, 1794): 2009

- Coccinella septempunctata (Linnaeus, 1758): 2006, 2009

- Hippodamia variegata (Goeze, 1777): 2006, 2009 (Fig. 4)

- Nephus bipunctatus (Kugelann, 1794): 2006, 2009

- Propylaea quatuordecimpunctata (Linnaeus, 1758): 2006

- Psyllobora vigintiduopunctata (Linnaeus, 1758): 2006

- Rhizobius lophantae (Blaisdell. 1892): 2006

- Scymnus apetzi Mulsant, 1846: 2006, 2009

- Scymnus interruptus (Goeze, 1777): 2006, 2009

- Scymnus mediterraneus Iablokoff-Khnzorian, 1972: 2006, 2009

- Scymnus subvillosus (Goeze, 1777): 2006, 2009

- Scymnus suturalis Thunberg, 1795: 2006, 2009

- Stethorus punctillum Weise, 1891: 2006, 2009

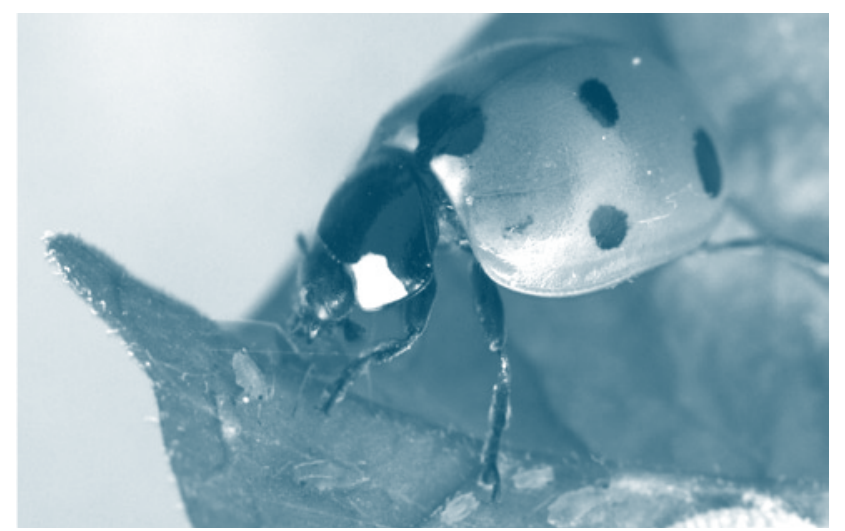

Figura 4. Hippodamia variegata depredando pulgón. Foto J. Ordónez

\section{PARASITOIDES}

\section{HYMENOPTERA}

Aphelinidae

- Aphelinus semiflavus Howard, 1908: 2006, 2009

Braconidae: Aphidiinae

- Adialytus salicaphis (Fitch, 1855): 2009

- Aphidius colemani Viereck, 1912: 2006, 2009

- Aphidius ervi Haliday 1834: 2006, 2009

- Aphidius funebris (Mackauer, 1961): 2006

- Aphidius matricariae Haliday, 1834: 2006, 2009

- Aphidius rhopalosiphi De Stefani-Perez, 1902: 2006

- Aphidius sonchi Marshall, 1896: 2006

- Aphidius uzbekistanikus Luzhetzki, 1960: 2006

- Diaretiella rapae (M’lntosh, 1855): 2006, 2009

- Ephedrus persicae Froggatt, 1904: 2006

- Lysiphlebus fabarum Marshall, 1896: 2006, 2009

- Lysiphlebus testaceipes (Cresson, 1880): 2006, 2009

- Praon abjectum (Haliday, 1833): 2006, 2009

- Praon volucre (Haliday, 1833): 2006, 2009 (Fig. 5)

- Praon yomenae Takada, 1968: 2009

- Trioxys angelicae (Haliday, 1833): 2006, 2009

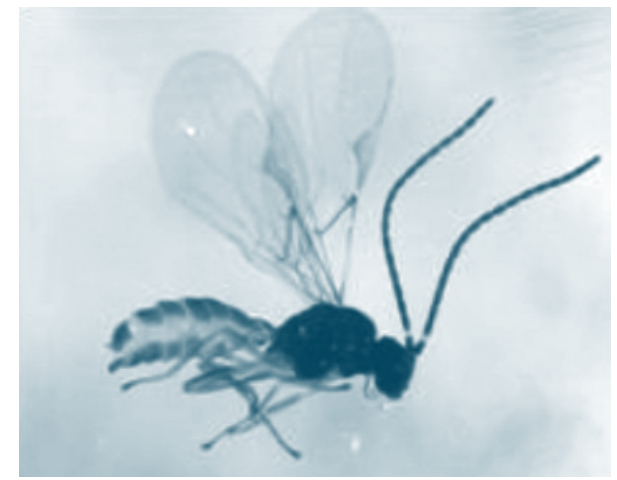

Figura 5. Praon volucre. Foto J. Quinto 


\section{DISCUSIÓN}

Las 48 especies registradas en este estudio ponen de manifiesto que los agroecosistemas mediterráneos ibéricos presentan una rica fauna autóctona de enemigos naturales de plagas de hemípteros esternorrincos, tanto depredadores como parasitoides. Estas comunidades de enemigos naturales están presentes a lo largo del ciclo de cultivo, lo que implica que diferentes especies de enemigos naturales se vayan sucediendo en los cultivos, permitiendo en ocasiones su establecimiento y permanencia en los mismos. Asimismo, la alta riqueza de especies de enemigos naturales registrada representa un importante factor en el control natural de las poblaciones de insectos plaga que inciden en los cultivos que componen este heterogéneo agroecosistema.

La integración de las diferentes estrategias de control dirigidas a potenciar las poblaciones de los enemigos naturales autóctonos a partir del manejo del hábitat, pueden aumentar la efectividad de este tipo de control biológico y disminuir el número de aplicaciones de fitosanitarios, minimizando los aspectos negativos que se derivan de su uso.

El conocimiento de la alta biodiversidad y abundancia de enemigos naturales autóctonos estudiados, indican la importancia de la conservación de zonas agrícolas formadas por un mosaico diversificado de cultivos separados por márgenes de vegetación herbácea silvestre o arvense (vegetación espontánea asociada a los cultivos), donde además de representar un recurso trófico suplementario para estos insectos, también puede servir de área de refugio para las diferentes fases de su ciclo de desarrollo. Por lo tanto, todas las acciones encaminadas a mejorar, diversificar y conservar el hábitat, contribuirán a potenciar el buen estado de las poblaciones de estos insectos que, sin a penas percatarnos, están siendo nuestros más eficaces aliados en la lucha contra las plagas.

\section{BIBLIOGRAFIA}

BERNDT, L.A., WRATTEN, S.D., 2005. Effects of alyssum flowers on the longevity, fecundity, and sex ratio of the leafroller parasitoid Dolichogenidea tasmanica. Biological Control, 32, 65-69

BLACKMAN, R.L. \& EASTOP, V.F., 2000. Aphids on the world's crop. An identification and information guide. John Wiley and Sons, 466p.

COLLEY, M.R., LUNA, J.M., 2000. Relative attractiveness of potential beneficial insectary plants to aphidophagous hoverflies (Diptera: Syrphidae). Environmental Entomology, 20 (5), 1054-1059

LANDIS, D.A., WRATTEN, S.D., SEDCOLE, J.R., 2006. Habitat management to conserve natural enemies of arthropod pest in agriculture. Annual Review of Entomology, 45, $175-201$

ÖLMEZ, S., ULUSOY, M.R., 2003. A Survey of Aphid Parasitoids (Hymenoptera: Braconidae: Aphidiinae) in Diyarbakir, Turkey. Phytoparasitica 31 (5), 524-528

PENNACCHIO, E., 1989. The Italian species of the genus Aphidius Nees (Hymenoptera, Braconidae, Aphidiinae). Boll. Lab. Ent. Agr. Filippo Silvestri 46: 75-106

PLAZA, E., 1979. Clave para la identificación de los géneros y catálogo de las especies españolas peninsulares y baleáricas de Coccinellidae. Graellsia, 42: 19-45.

REMAUDIÈRE, G. \& SECO, M.V., 1990. Claves de pulgones alados de la región mediterránea. Rústica. 2 Vol. 110p.

STARY, P., 1976. Aphid parasites (Hymenoptera, Aphidiidae) of the Mediterraneus Area. Dr. W. Junk, B.V. Publishers, The Hague. Trans Czechols Acad Sci, Ser. Math. Nat. Sci 86(2): 1-95

STERN, V.,M., SMITH, R.F., VAN DER BOSCH, R., HAGEN, K.S., 1959. The integrated control concept. Hilgardia 29, 81-101

VAN DER VEEN, M., 2004. Hoverflies of Northwest Europe: identification keys to the Syrphidae. KNNV Publishing, Utrecht

\section{AGRADECIMIENTOS}

A los Doctores J.M. Michelena, R. Jiménez y M.J. Verdú por su inestimable ayuda en la identificación del material. A los agricultores del Pilar de la Horadada que se prestaron a colaborar con nosotros y que confían en el control biológico. 